ORNL/TM-2005/113

\title{
Ontology-enriched Visualization of Human Anatomy
}

\section{May 2005}

Prepared by

\section{L.C. Pouchard}

S. P. Dickson

Oak Ridge National Laboratory

Supported by the Laboratory Director's Research and Development Fund S03-26 


\section{DOCUMENT AVAILABILITY}

Reports produced after January 1, 1996, are generally available free via the U.S. Department of Energy (DOE) Information Bridge:

Web site: http://www.osti.gov/bridge

Reports produced before January 1, 1996, may be purchased by members of the public from the following source:

National Technical Information Service

5285 Port Royal Road

Springfield, VA 22161

Telephone: 703-605-6000 (1-800-553-6847)

TDD: $703-487-4639$

Fax: 703-605-6900

E-mail: info@ntis.fedworld.gov

Web site: http://www.ntis.gov/support/ordernowabout.htm

Reports are available to DOE employees, DOE contractors, Energy Technology Data Exchange (ETDE) representatives, and International Nuclear Information System (INIS) representatives from the following source:

Office of Scientific and Technical Information

P.O. Box 62

Oak Ridge, TN 37831

Telephone: 865-576-8401

Fax: 865-576-5728

E-mail: reports@adonis.osti.gov

Web site: http://www.osti.gov/contact.html

This report was prepared as an account of work sponsored by an agency of the United States Government. Neither the United States government nor any agency thereof, nor any of their employees, makes any warranty, express or implied, or assumes any legal liability or responsibility for the accuracy, completeness, or usefulness of any information, apparatus, product, or process disclosed, or represents that its use would not infringe privately owned rights. Reference herein to any specific commercial product, process, or service by trade name, trademark, manufacturer, or otherwise, does not necessarily constitute or imply its endorsement, recommendation, or favoring by the United States Government or any agency thereof. The views and opinions of authors expressed herein do not necessarily state or reflect those of the United States Government or any agency thereof. 


\title{
Ontology-enriched Visualization of Human Anatomy
}

\author{
L.C. Pouchard and S. P. Dickson \\ Computer Science and Mathematics Division \\ Oak Ridge National Laboratory
}

Date Published: May 2005

Prepared by

OAK RIDGE NATIONAL LABORATORY

P. O. Box 2008

Oak Ridge, Tennessee 37831-6285

managed by

UT-Battelle, LLC

for the

U.S. DEPARTMENT OF ENERGY

under contract DE-AC05-00OR22725 


\section{TABLE OF CONTENTS}

\section{Page}

LIST OF FIGURES

iv

ABSTRACT

V

1. INTRODUCTION 1

2. TECHNICAL APPROACH 2

3. RESULTS 5

3.1. TESTING SEARCH MECHANISMS

3.2. ANALYSIS AND DISCUSSION OF SEARCH MECHANISMS 6

3.3. VISUALIZATION

4. SUMMARY 10

REFERENCES 11 


\section{List of Figures}

Page

Figure 1: Foot volumetric data. $\quad 2$

Figure 2: First metatarsal bone in the Foundational Model Anatomy. 3

Figure 3: Nodes and edges for FMA entities in the parts of the talus. 5

Figure 4: Maya ${ }^{\mathrm{TM}}$ search plug-ins. $\quad 8$

Figure 5: The Hotbox: knowledge-guided user interface. 8

Figure 6: 3-D rendering of muscles attached to the metatarsal bones. 9 


\begin{abstract}
The project focuses on the problem of presenting a human anatomical 3D model associated with other types of human systemic information ranging from physiological to anatomical information while navigating the 3D model. We propose a solution that integrates a visual 3D interface and navigation features with the display of structured information contained in an ontology of anatomy where the structures of the human body are formally and semantically linked. The displayed and annotated anatomy serves as a visual entry point into a patient's anatomy, medical indicators and other information. The ontology of medical information provides labeling to the highlighted anatomical parts in the 3D display. Because of the logical organization and links between anatomical objects found in the ontology and associated 3D model, the analysis of a structure by a physician is greatly enhanced. Navigation within the 3D visualization and between this visualization and objects representing anatomical concepts within the model is also featured.
\end{abstract}




\section{INTRODUCTION}

As the use of computer modeling spreads in medical research, smarter models of the human anatomy are needed in a broad range of applications, including clinical diagnosis, surgical planning [1], cancer treatment using radiation therapy [2], and other applications. In silico models constructed in computerized simulation are used to determine the effects of parameter variations and compare between actual clinical data and simulation results. Static models of human anatomy in 2D slices are now available to clinicians and researchers for visualization, diagnosis, measurements and other purposes. From scans such as X-ray Computed Tomography (CT) or Magnetic Resonance Imagery (MRI ) volumetric reconstructions of the human body are created. However, the volumetric reconstructions have not been used for 3D animations for scientific and medical purposes, in part due to computational complexity and resource requirements.

In addition, researchers, clinicians, and practitioners who analyze computerized segments of the anatomy do not have the ability to integrate different types of medical data. There is also a lack of easy-to-use capabilities to facilitate analysis and increase accuracy. At the time of data analysis and visualization, scientists must rely on their own mental models to integrate into a coherent whole information provided by different systems and represented in several media: multi-resolution imaging, property measurements (physiological, mechanical, etc.), standardized annotations of anatomy, characterized links between parts of the anatomy, sensor data and others.

Faced with an explosion of parameters to consider when making decisions, researchers and practitioners need new computerized environments where various media types and tools are seamlessly integrated and easily manipulated at the point of service [3]. Tools for analysis and diagnosis guidance may be critical for the quick assessment of an emergency situation. This is particularly true when split-second assessment of an injury is required, for instance by paramedics in the field of battle [4].

Based on this ORNL LDRD S03-26 research a proposal to the DARPA Virtual Soldier Program (VSP) Phase I [5] has been selected and the team invited to participate in the VSP under sponsorship of the United States Army Medical Research and Materiel Command (USAMRMC) (DOE Proposal Number 1868-HH48-X1). Results described below were innovative and proved highly applicable to the work in the VSP. The work reported here focused on visualizing 3D anatomy and annotating it with structured information contained in a knowledge base. Search methodologies for an ontology-based 3D system were also explored. 


\section{TECHNICAL APPROACH}

Animated human models have been developed in the cinematic industry with an emphasis on good appearance rather than accuracy of representation. The level of detail and accuracy of 3D models appropriate for the needs of scientific representation is lacking in state-of-the-art 3D anatomical representation from the industry. Accurately and precisely identify anatomical parts with 3D volumetric coordinates at a level of precision not achieved previously, visualizing parts of the anatomy with this new level, and easy-to-use navigation capabilities are investigated here. The foot is the chosen structure for visualization as an example of an anatomical part with a high concentration of interacting small muscles and bones, and variety of tissue. Maya ${ }^{\mathrm{TM}}$, [6] a highly flexible environment with solid community support and numerous extensions is the development environment for 3D animation utilized here (Figure 1).

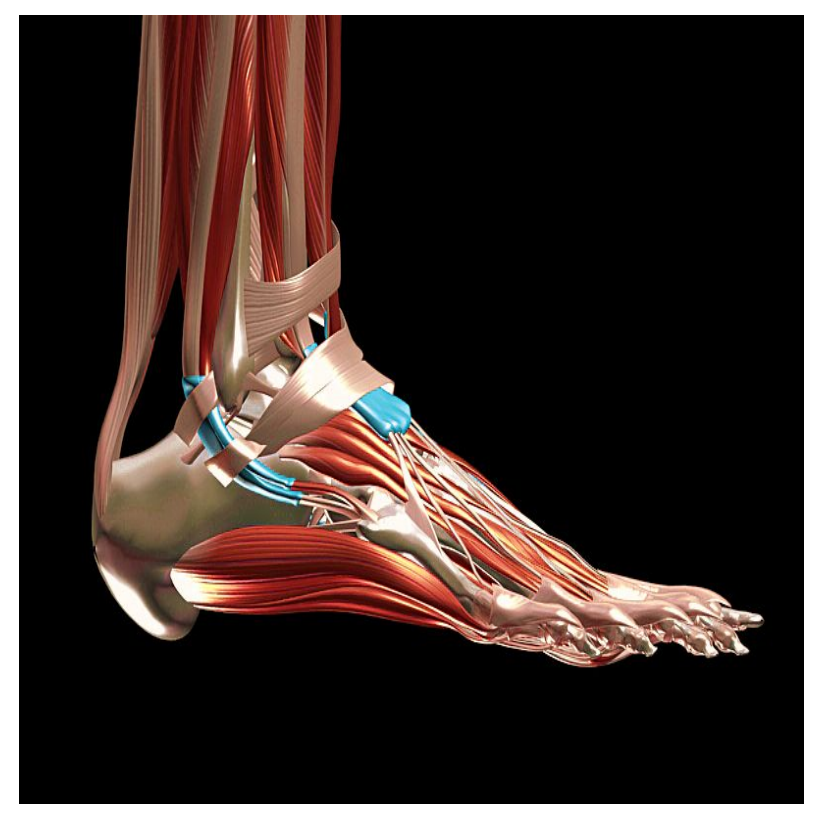

Figure 1: Foot volumetric data.

A multi-level information infrastructure is also investigated. This infrastructure permits associating each modeled part of the anatomy and its corresponding geometric coordinates to a network of textual information and properties contained in an ontology. An ontology is a declarative, symbolic model that formalizes (anatomical) concepts, terminology, and abstract relationships between these concepts. It also contains logical rules on how relationships may combine concepts. All items in an ontology are called frames. An ontology is best understood as a semantic graph structured with links as edges and semantic definitions as nodes. The information may include text, parts of speech, concepts, images, or mathematical coordinates that are linked with each other by logical relationships described in rules. It is by design human and machine-readable, non-exhaustive and able to accommodate new types of information and concepts thanks to its formal specifications when new discoveries are being made. Some 
purposes of ontologies include creating standards on a particular topic, declaring machinereadable specifications, and re-use of components. The Foundational Model Anatomy (FMA) [7] is the domain ontology we use (Figure 2).

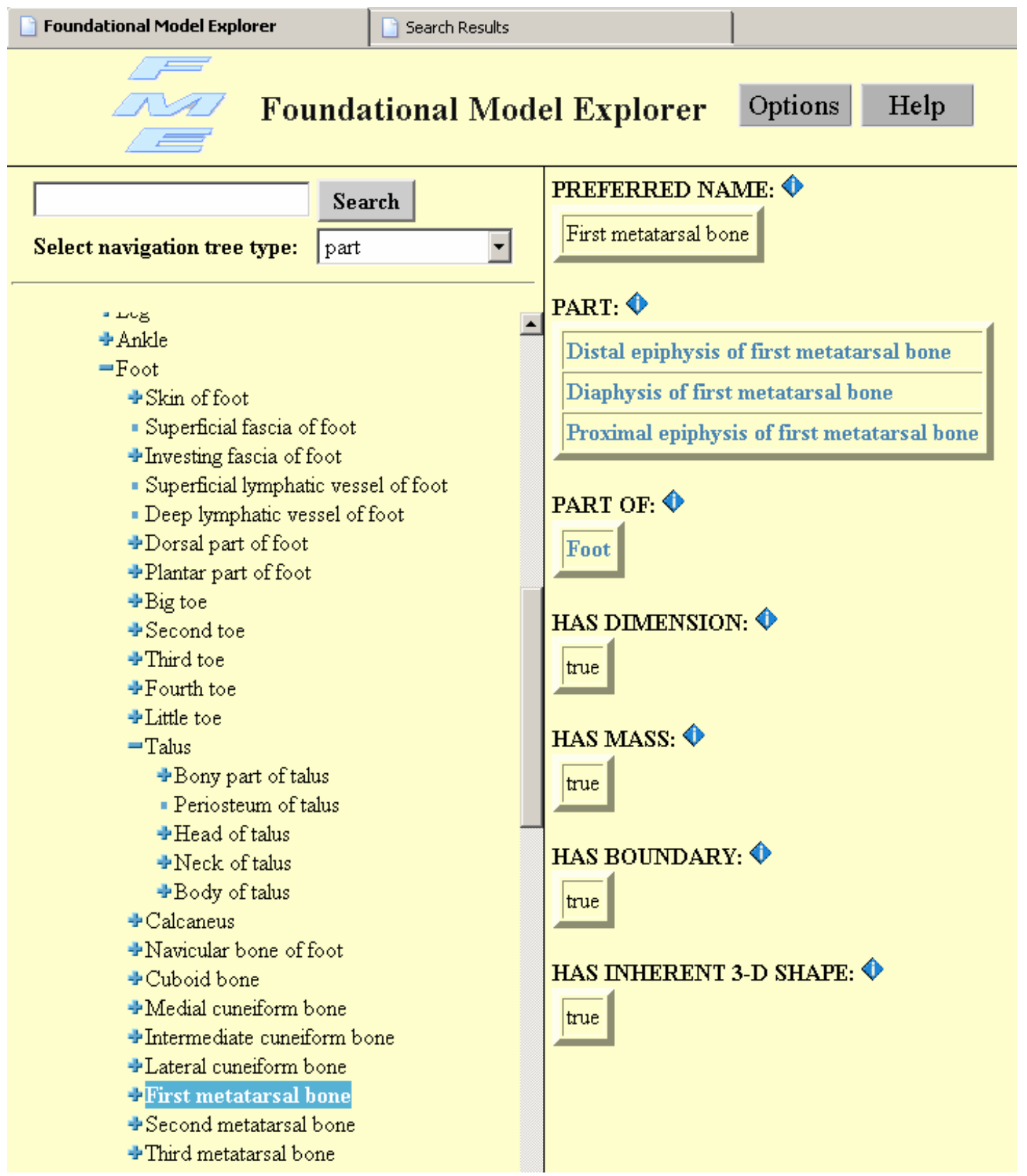

Figure 2: First metatarsal bone in the FMA.

The FMA was developed at the University of Washington and contains approximately 100,000 terms representing 70,000 concepts, 120 logical relationships iterated over a million times between the terms. As an example, in the FMA, tissues are related to each other through abstract relationships such as "has boundary," "is a tributary of," "is contiguous to," and many others. 
The FMA is based on the Terminologia Anatomica. In addition, it also includes physiological and some cell-related information that is characterized with relationships to anatomical entities.

A medical researcher or scientist navigating the abstract relationships of the FMA in a browser sees the connections between entities based on the FMA relationships and may predict interactions between seemingly unrelated entities. By pulling the 3D coordinates corresponding to FMA entities into a visualized model, our system enables him or her to better analyze hypotheses and compare individual subject data with ranges of average data. The researcher is also able to display detailed numerical and textual information associated with an anatomical structure thanks to a link to the corresponding FMA entity. The resulting visualization in animated 3D rendering of the anatomy and annotated with information contained in the FMA enhances the analysis of a patient's anatomy.

The FMA is represented in Protégé [10], an open source, highly sophisticated set of tools for ontological engineering developed at the Stanford Medical Informatics Institute. Features include a text browser, a diagramming tool, and a knowledge acquisition and editing tool. Protégé is entirely designed using Sun Java ${ }^{\mathrm{TM}}$ technology. One of its features is the possibility of extending and customizing the system with plug-ins without substantially modifying the code. A plug-in is a self-contained collection of java objects that adds functionality to the representation tool through a simple java API. Access to the Foundational Model through the Web is also a feature.

Building structured knowledge in 3D graphical representation serves as a basis for designing querying mechanisms to the visualization. Single slice (2-D) volume images are aligned with the 3D Digital Model and the Model scaled to match the image. The 3D Digital Model is articulated to match a whole-body surface scan and geometrical entities are selected within the Digital Model. This provides a "Deep Voxel" feature -- i.e., a zooming-in and out of the model - that triggers -referencing to the corresponding entity in the ontology. Cross-referencing the visual model and the ontological model is obtained thanks to a combination of the entity-naming strategy enforced in the construction of the Digital Model and the design of the plug-ins [12].

The approach also includes designing search and retrieval mechanisms that are both appropriate to the visualization environment and return accurate and meaningful answers for the scientist. A movement of the computer mouse within visualization translates into a computerized query to the FMA. Two query mechanisms associated with the FMA are investigated: a query through the Protégé interface, and direct logic-based queries through a Web-interface. Query and return formats for the visual interface were also investigated because the format of a query and its display affect the number of nodes traversed in the ontology. 


\section{RESULTS}

The results found here are based on heuristics and the researchers' experience, and do not include metrics. They are still valuable because they guide the choice of computer architecture for integerating visual, textual, and logical information. This research proved necessary for developing middleware architecture for the Virtual Soldier Program and is expected to continue under the U.S. Army Medical Research Command sponsorship. The system also contains the facilities for posing the Digital Anatomist model, creating scripted animation of the character and a limited degree of simulation. The simulation features of the animation system may be exploited primarily to gain a naturalistic response of soft tissues (muscles and connective tissue) in response to articulation of the skeleton to which they are attached.

\subsection{SEARCH MECHANISMS AND INTERFACES.}

The FMA is available through a Web interface that uses the Foundational Model Explorer [8] as a browser tool for navigating the FMA. A Search for the text string "first metatarsal bone" entered in the Search box and a check on the "subclass" for the navigation tree type of query shows the results of a query for the first metatarsal bone (Figure 2): it is a subclass of metatarsal bone; it has three parts; it is a part of the foot, and a regional part of the skeleton of the foot; it has a boundary, an inherent 3D shape and no synonym. (By contrast, blood is part of the hematopoietic system and is a subclass of body substances; it has boundary but no inherent 3D shape.)

The results of a two-level query for the talus (heel) are shown in Figure 3. It displays first level nodes on the hierarchical "part-of" concepts that constitute the talus (green circles) and some second level nodes (orange circles). In the FMA the foot is a "region" of the body. The talus is a subclass of tarsal bone, itself a subclass of short bones (not shown here). This example illustrates the anatomical relationships (part-of, subclass) in a structured ontology of what is commonly thought of as a part of the body. The FMA has been used for educational purposes, but our research shows that it can also be used as a diagnostic tool when integrated into a visualization environment.

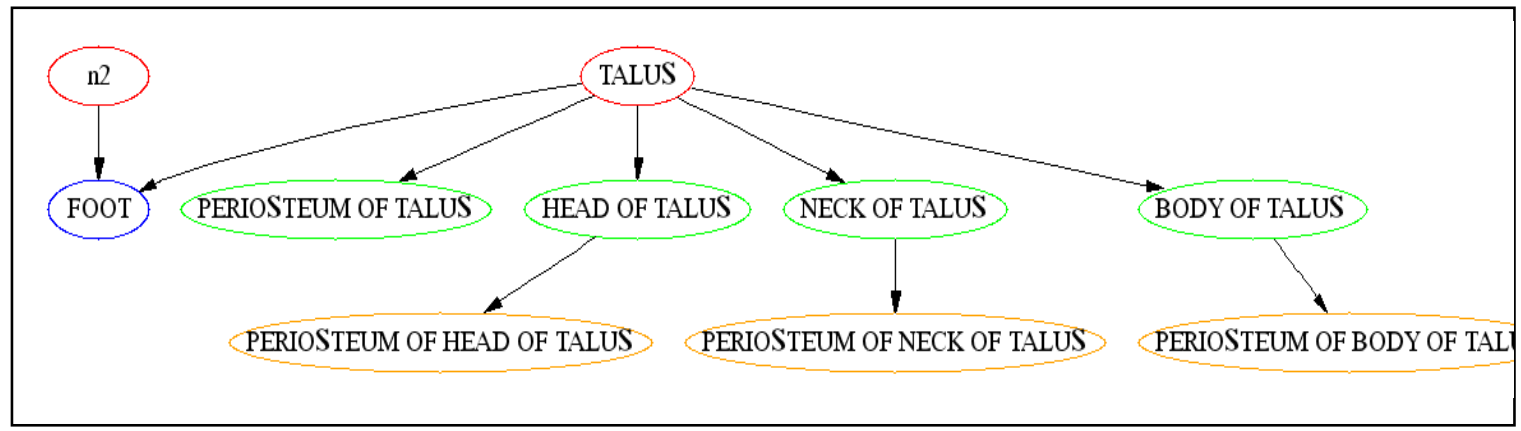

Figure 3: Nodes and edges for FMA entities in the parts of the talus. 
Searches in ontology are more complex than searches in a database because ontologies allow broader queries where certains parts of the query are unknown simultaneously with more precise definitions (and thus are more flexible). One key feature of ontologies is the possibility to perform searches using rules rather than matching cell content (keywords or other data) as in a relational database. As exemplified above for the FMA, a search on a string can be specified as "string as part of" or "string as subclass" or "string as regional part."

Search methodologies and the software that enable searches to the ontology by mouse-over 3D modeling effectively implement the integration between the ontology and the visualization environment. The Ontology Querying Agent for the Foundational Model of Anatomy (OQAFMA), a query system developed for the FMA [9], and the Protégé query mechanisms were tested. The query language and data formats for displaying the returns into a visualization environment are distinctive features for each mechanism and produce different methods for constructing a query. It is important to consider the complexity of formulating a query in regard with the user-physician. A knowledge of the FMA relationships in the ontology is also required. Keeping this in mind, queries on the "part_of" and "has_boundary" were constructed and embedded in the environment (see example below).

\subsection{ANALYSIS AND DISCUSSION OF SEARCH MECHANISMS}

OQAFMA is based on STRUQL, a modified version of SQL to include rule-based searches. For a given query OQAFMA traverses the entire tree of the FMA, and returns the results, not only at the first level of the FMA tree where strings are found, but also at all branches of the tree down to the last leaf satisfying the criteria. This is called "bringing closure" to a search. By contrast, a search using Protégé search mechanisms does not bring closure as it returns only the first level of nodes in the tree. To obtain closure with Protégé, it would be necessary to parse each element returned by a search and perform new searches based on relationships found between these elements until a search returns no result. For this reason, OQFMA search mechanisms were chosen over Protégé searches. OQAFMA is also a more economical and elegant solution.

Due to the large number of possible iterations (over one million) (i.e. meaningful combinations) in the FMA, the search space must be reduced. A good rule of thumb is to reduce the number and depth of queries according to the level of detail that can be displayed in the visualization. In the case of visualization of human anatomy, two levels of queries in the hierarchy of the FMA are adequate because details provided at more granular level are not represented.

The results of OQAFMA queries are formatted as XML documents directly by the OQAFMA server, which are then processed for the visualization environment. However, XML cannot easily represent a tree hierarchy, non-hierarchical relationships, and rules for constraining the use of elements. The query below illustrates an OQAFMA query written in the Knowledge Interchange Language (KIF) [11] for the parts of the Talus. Currently, KIF is a draft proposed American National Standard NCITS.T2/98-004 
Example: Search for "talus" using OQAFMA.

\section{Query:}

\section{WHERE}

X->":NAME"->"talus",

$\mathrm{X}->$ "part"+->Y,

$\mathrm{Y}->$ ":NAME"->Parts

CREATE

TheTalus(Parts)

\section{Results:}

$<$ results $>$

$<$ Talus $>$

$<$ Parts $>$ Head of talus $<$ /Parts $>$

$<$ Talus $>$

$<$ Talus $>$

$<$ Parts $>$ Neck of talus $<$ /Parts $>$

$<$ Talus $>$

$<$ Talus $>$

$<$ Parts $>$ Body of talus $<$ /Parts $>$

$<$ Talus $>$

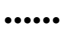

$<$ Talus $>$

$<$ Parts $>$ Periosteum of head of talus $</$ Parts $>$

$<$ Talus $>$

$<$ Talus $>$

$<$ Parts $>$ Periosteum of neck of talus $<$ Parts $>$

$<$ Talus $>$

$<$ Talus $>$

$<$ Parts $>$ Periosteum of body of talus $<$ Parts $>$

$<$ Talus $>$

-....

$<$ results $>$

When contrasted with Figure 3, one notices that head, neck and body of talus are at the same XML element level as periosteum of head, neck and body. The hierarchy exemplified in Figure 3 has been flattened.

\subsection{VISUALIZATION}

The system is prototyped as a collection of Maya plugins (Figure 4). DH_SelectEntity is declared in Maya ${ }^{\mathrm{TM}}$ as a global procedure which is called when the user selects an entity from the Digital Human customized Marking Menu. DH_SelectEntity maps from the Foundational Model of Anatomy entity name to the name of the corresponding geometry node in the Maya Scene Graph (Directed Acyclic Graph), then selects the appropriate geometry node for focus. The global procedure DH_SelectionChange is a custom Maya ${ }^{\mathrm{TM}}$ Embedded Language command executed upon a change of the selection list. DH_SelectionChange maps from the selected geometrical entity in the Maya Scene Graph to the corresponding ontological name for the node. 
DH_SelectionChange builds and sends the query to OQFMA and collects the results. DH_SelectionChange then edits the marking menu to reflect the relational information relevant to the current focus context. The 3D volume rendering of muscles attached to the metatarsal bones in Maya ${ }^{\mathrm{TM}}$ is displayed in Figure 6.

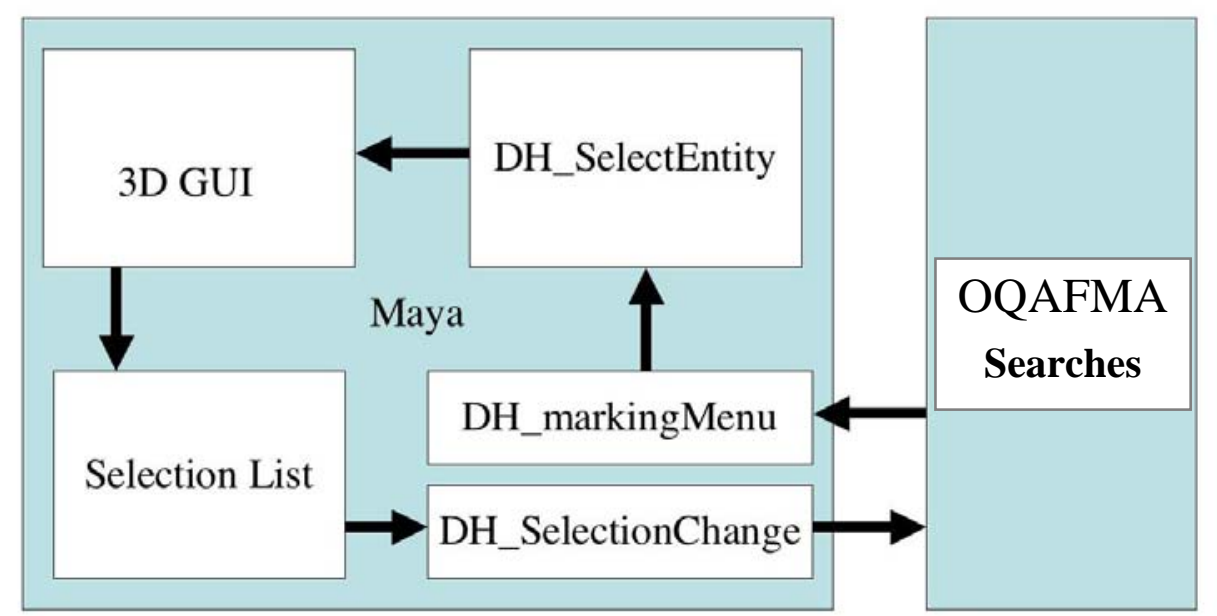

Figure 4: Maya ${ }^{\mathrm{TM}}$ search plug-ins.

The OQAFMA query results for a query on the heart are displayed in the Hotbox, a knowledgeguided 3-D user interface designed for the Virtual Soldier Project (Figure 5). The Hotbox shows a search on the Parts relationship in the FMA. Implementation of the Hotbox is pursued within the scope of the VSP project [13].

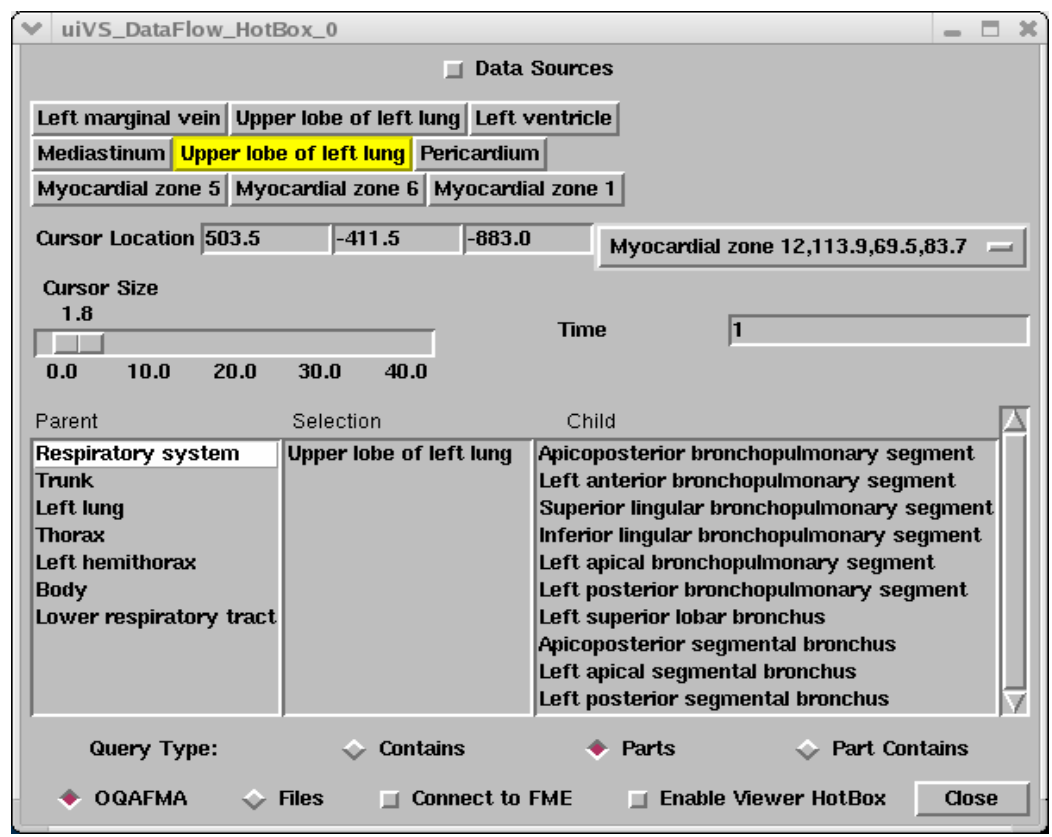

Figure 5: the Hotbox: knowledge-guided user interface. 


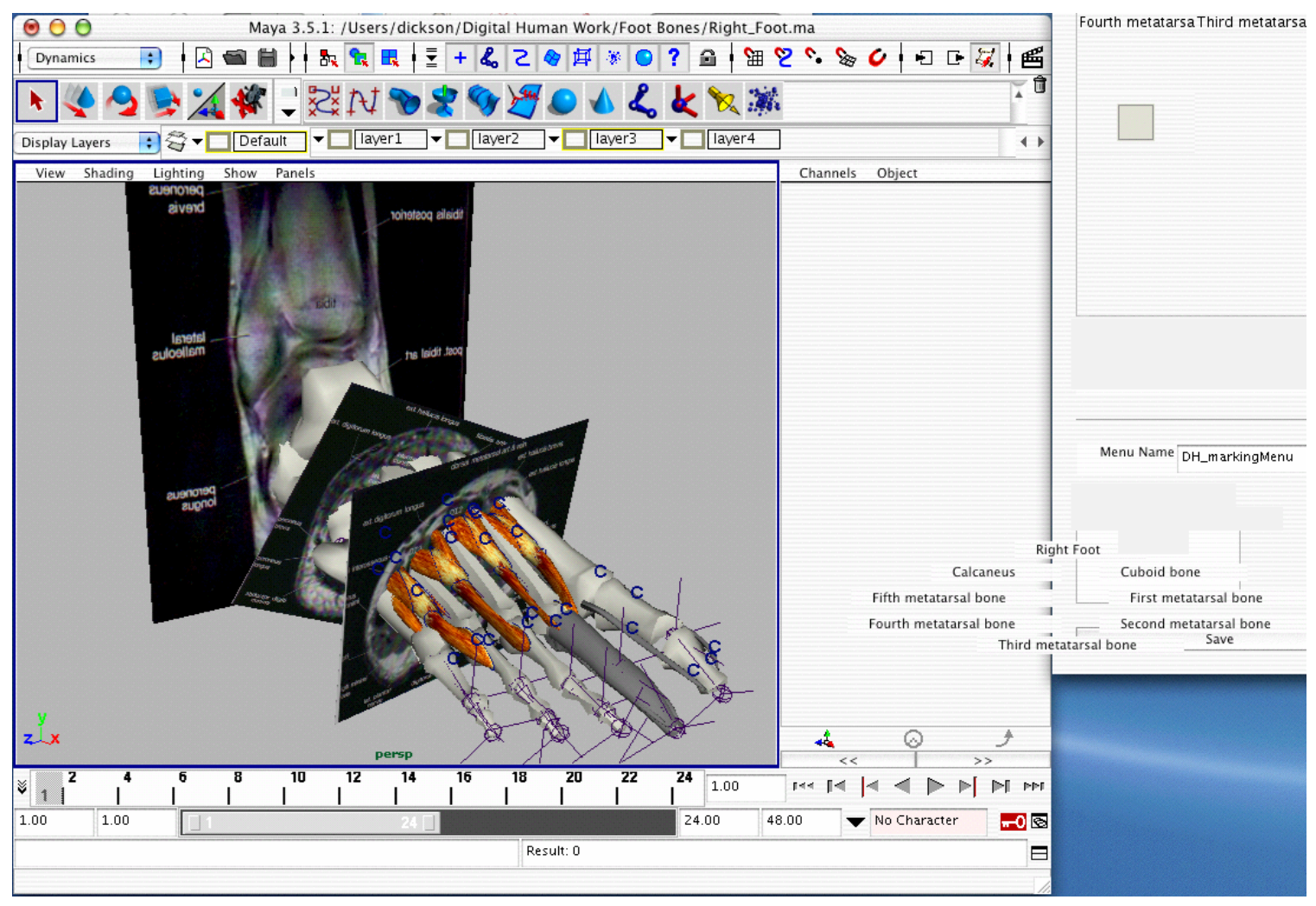

Figure 6: 3-D rendering of muscles attached to the metatarsal bones. 


\section{SUMMARY}

The FMA and its various access interfaces was obtained from the University of Washington (UW) and resides on an ORNL server. A collaboration with the University of Washington was formed and is still very active at the time of this writing. At this time, ORNL is not expected to become a mirror site for the FMA. Access to the FMA data and ORNL visualization architecture by team members of the VSP has been provided and used during Phase I of the VSP within the framework of DOE proposal 1868-HH48-X1. Search mechanisms using OQAFMA have been tested for seamless access by computer applications and a Maya - Protégé API was designed. The results of this work were later incorporated into the VSP HotBox, an ontology-based 3D visualization application using an open source visualization framework. The Hotbox performs searches in the FMA using the OQAFMA search mechanisms accessed by a Web service. Further research directions include ontology representation systems and visualization, and ontology search languages. 


\section{REFERENCES}

[1] Westwood, J.D., et al., editors, Medicine Meets Virtual Reality 2000, IOS Press, Amsterdam, (2000).

[2] Kahn, F.M., The Physics of Radiation Therapy, $2^{\text {nd }}$ Ed., Williams \& Wilkins, Baltimore, (1994).

[3] Satava, R. M., "Cybersurgeon: Advanced simulation technologies for surgical education," Medical Simulation and Training, 1:6-9 (1996).

[4] Satava, R.M., "Medical Virtual Reality," Medicine Meets Virtual Reality 4: Health Care in the Information Age - Future Tools for Transforming Medicine, IOS Press, Amsterdam, pp. 100-105 (1996).

[5] The Virtual Soldier Program. http://www.virtualsoldier.net.

[6] The Maya Family. (C2000-2003 Alias Systems, a division of Silicon Graphics Limited. All rights reserved.

[7] Rosse, C., Shapiro, L.G., Brinkley, J.F., The Digital Anatomist Foundation Model: Principles for Defining and Structuring its Concept Domain, J Am Med Inform Assoc. American Medical Informatics Association (AMIA) 1998 Symp. Suppl. 1998:820-824.

http://sig.biostr.washington.edu/projects/fm/index.html.

[8] FMA. http://sig.biostr.washington.edu/projects/fm/index.html.

[9] OQAFMA, Forthcoming paper by Peter Mork, http://quad.biostr.washington.edu/noqafma/index.html.

[10] Protégé, http://protege.stanford.edu/.

[11] Genesereth, M. and R.E. Fikes, "Knowledge Interchange Format, Version 3.0. Reference Manual,” Technical Report Logic-92-1, Computer Science Department, Stanford University, Stanford, CA, January 1992.

[12] Dickson, S., Pouchard, L. C., and R.D. Ward, “Integration of an Ontology and a 3D Animation Model for the Digital Human Project,” Medicine Meets Virtual Reality 11, Newport Beach, CA, USA, January 22-25, 2003.

[13] R.D. Ward, Pouchard, L. C., and Dickson, S., "Linking Human Anatomy to Knowledge bases: A Visual Front-end to Electronic Medical Records,” Medicine Meets Virtual Reality 13, Long Beach, CA, USA, January 26-29, 2005. 


\section{DISTRIBUTION LIST}

\section{Electronic Distribution}

1. J. A. Nichols

2. S. Dickson

3. L. C. Pouchard

4. T. Zacharia

5. ORNL Central Research Library

6. ORNL Laboratory Records - RC

7-8. ORNL Laboratory Records - OSTI 
The submitted manuscript has been authored by the US Department of Energy, Office of Science of the Oak Ridge National Laboratory, managed for the U. S. DOE by UTBattelle, LLC, under contract No. DE-AC05-000R22725. Accordingly, the U.S. Government retains a non-exclusive, royalty-free license to publish or reproduce the published form of this contribution, or allow others to do so, for U.S. Government purposes. 